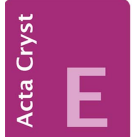
COMMUNICATIONS

ISSN 2056-9890

\section{Crystal structure of the enol form of mesotrione: a benzoylcyclohexanedione herbicide}

\author{
Gihaeng Kang, Jineun Kim, ${ }^{*}$ Hyunjin Park and Tae Ho \\ Kim*
}

Department of Chemistry and Research Institute of Natural Sciences, Gyeongsang National University, Jinju 660-701, Republic of Korea. ${ }^{*}$ Correspondence e-mail:

thkim@gnu.ac.kr, jekim@gnu.ac.kr

Received 30 June 2015; accepted 2 July 2015

Edited by H. Stoeckli-Evans, University of Neuchâtel, Switzerland

The title compound [systematic name: 3-hydroxy-2-(4-methylsulfonyl-2-nitrobenzoyl)cyclohex-2-enone], $\mathrm{C}_{14} \mathrm{H}_{13} \mathrm{NO}_{7} \mathrm{~S}$, is the enol form of a benzoylcyclohexanedione herbicide. As a result of this tautomerization, there is intramolecular $\mathrm{O}-$ $\mathrm{H} \cdots \mathrm{O}$ hydrogen bond enclosing an $S(6)$ ring motif. The cyclohexene ring has an envelope conformation, with the central $\mathrm{CH}_{2} \mathrm{C}$ atom as the flap. Its mean plane is inclined to the benzene ring by $87.46(8)^{\circ}$. In the crystal, molecules are linked by a series of $\mathrm{C}-\mathrm{H} \cdots \mathrm{O}$ hydrogen bonds, forming a three-dimensional framework.

Keywords: crystal structure; tautomerization; enol form; intramolecular $\mathrm{O}-\mathrm{H}$... $\mathrm{O}$ hydrogen bond.

CCDC reference: 1410192

\section{Related literature}

For information on the herbicidal properties of the title compound, see: Mitchell et al. (2001). For related crystal structures, see: Eftekhari-Sis et al. (2012); Liu \& Tang (2012).<smiles>CS(=O)(=O)c1ccc(C(=O)C2=C(O)CCCC2=O)c([N+](=O)[O-])c1</smiles>

\section{Experimental}

2.1. Crystal data

$\mathrm{C}_{14} \mathrm{H}_{13} \mathrm{NO}_{7} \mathrm{~S}$

$M_{r}=339.31$

Monoclinic, $P 2_{1} / c$

$a=10.4208(2) \AA$

$b=11.2525(3) \AA$

$c=12.3550(3) \AA$

$\beta=95.370(1)^{\circ}$

$V=1442.39(6) \AA^{3}$

$Z=4$

Mo $K \alpha$ radiation

$\mu=0.26 \mathrm{~mm}^{-1}$

$T=173 \mathrm{~K}$

$0.43 \times 0.30 \times 0.20 \mathrm{~mm}$

\subsection{Data collection}

Bruker APEXII CCD diffractometer

Absorption correction: multi-scan (SADABS; Bruker, 2009)

$T_{\text {min }}=0.895, T_{\max }=0.949$ 2828 independent reflections 2572 reflections with $I>2 \sigma(I)$ $R_{\text {int }}=0.025$

\subsection{Refinement}

$R\left[F^{2}>2 \sigma\left(F^{2}\right)\right]=0.036$

$w R\left(F^{2}\right)=0.097$

$S=1.04$

2828 reflections

213 parameters

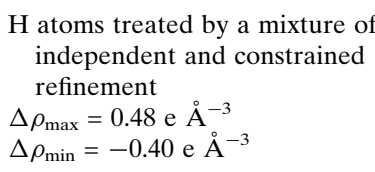

$\mathrm{H}$ atoms treated by a mixture of independent and constrained refinement

$\Delta \rho_{\max }=0.48$ e $\AA^{-3}$

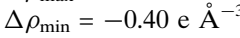

Table 1

Hydrogen-bond geometry $\left(\AA{ }^{\circ}\right)$.

\begin{tabular}{lllll}
\hline$D-\mathrm{H} \cdots A$ & $D-\mathrm{H}$ & $\mathrm{H} \cdots A$ & $D \cdots A$ & $D-\mathrm{H} \cdots A$ \\
\hline O6-H6O $\cdots \mathrm{O} 5$ & $0.91(3)$ & $1.71(3)$ & $2.524(2)$ & $148(2)$ \\
$\mathrm{C} 1-\mathrm{H} 1 B \cdots \mathrm{O} 4^{\mathrm{i}}$ & 0.98 & 2.58 & $3.393(2)$ & 140 \\
$\mathrm{C} 1-\mathrm{H} 1 B \cdots 7^{\mathrm{ii}}$ & 0.98 & 2.58 & $3.265(2)$ & 127 \\
$\mathrm{C} 11-\mathrm{H} 11 A \cdots \mathrm{O} 3^{\mathrm{iii}}$ & 0.99 & 2.40 & $3.135(2)$ & 131 \\
\hline Symmetry codes: & $(\mathrm{i})$ & $-x+1,-y+1,-z+2 ;$ & (ii) $x,-y+\frac{1}{2}, z+\frac{1}{2} ;$ & (iii) \\
$-x+2, y-\frac{1}{2},-z+\frac{3}{2}$. & & & &
\end{tabular}

Data collection: APEX2 (Bruker, 2009); cell refinement: SAINT (Bruker, 2009); data reduction: $S A I N T$; $\operatorname{program}(\mathrm{s})$ used to solve structure: SHELXS97 (Sheldrick 2008); program(s) used to refine structure: SHELXL2013 (Sheldrick, 2015); molecular graphics: DIAMOND (Brandenburg, 2010); software used to prepare material for publication: SHELXTL (Sheldrick 2008).

\section{Acknowledgements}

This research was supported by the Basic Science Research Program through the National Research Foundation of Korea (NRF) funded by the Ministry of Education, Science and Technology (No. 2014R1A1A4A01009105).

Supporting information for this paper is available from the IUCr electronic archives (Reference: SU5166).

\section{References}

Brandenburg, K. (2010). DIAMOND. Crystal Impact GbR, Bonn, Germany. Bruker (2009). APEX2, SAINT and SADABS. Bruker AXS Inc., Madison, Wisconsin, USA.

Eftekhari-Sis, B., Mohajer, S. \& Büyükgüngör, O. (2012). Acta Cryst. E68, o2829.

Liu, W. \& Tang, L. (2012). Acta Cryst. E68, o2850. 
Mitchell, G., Bartlett, D. W., Fraser, T. E. M., Hawkes, T. R., Holt, D. C., Townson, J. K. \& Wichert, R. A. (2001). Pest. Manag. Sci. 57, 120-128.
Sheldrick, G. M. (2008). Acta Cryst. A64, 112-122.

Sheldrick, G. M. (2015). Acta Cryst. C71, 3-8. 


\section{supporting information}

Acta Cryst. (2015). E71, o548-o549 [https://doi.org/10.1107/S2056989015012803]

\section{Crystal structure of the enol form of mesotrione: a benzoylcyclohexanedione herbicide}

\section{Gihaeng Kang, Jineun Kim, Hyunjin Park and Tae Ho Kim}

\section{S1. Comment}

Mesotrione, [keto form systematic name: 2-(4-mesyl-2-nitrobenzoyl)cyclohexane-1,3-dione], is a benzoylcyclohexanedione herbicide and it has been developed for the selective pre- and post-emergence control of a wide range of broadleaved and grass weeds in maize (Mitchell et al., 2001). However, until now its crystal structure has not been reported.

The title compound crystallized in the enol form (Fig. 1 and Table 1), with an intramolecular O6- $\mathrm{H} 6 \mathrm{O} \cdots \mathrm{O} 5$ hydrogen bond embedded in an $S(6)$ ring. The cyclohexene ring has an envelope conformation with the central $\mathrm{CH}_{2} \mathrm{C}$-atom, $\mathrm{C} 12$, as the flap. Its mean plane is inclined to the benzene ring by $87.46(8)^{\circ}$.

All bond lengths and bond angles are normal and comparable to those observed in the crystal structures of similar compounds (Eftekhari-Sis et al., 2012; Liu et al., 2012).

In the crystal, molecules are linked by a series of $\mathrm{C}-\mathrm{H} \cdots \mathrm{O}$ hydrogen bonds forming a three-dimensional framework (Fig. 2 and Table 1).

\section{S2. Experimental}

The title compound was purchased from the Dr. Ehrenstorfer GmbH Company. Slow evaporation of a solution in $\mathrm{CH}_{3} \mathrm{CN}$ gave single crystals suitable for X-ray analysis.

\section{S3. Refinement}

The O-bound $\mathrm{H}$ atom was located in a difference Fourier map and freely refined [O-H $=0.91$ (3) $\AA$ ]. The $\mathrm{C}$-bound $\mathrm{H}$ atoms were positioned geometrically and refined using a riding model: $\mathrm{C}-\mathrm{H}=0.95-0.99 \backslash \% \mathrm{~A}$ with $U_{\text {iso }}(\mathrm{H})=1.5 U_{\text {eq }}(\mathrm{C})$ for methyl $\mathrm{H}$ atoms and

$1.2 U_{\mathrm{eq}}(\mathrm{C})$ for other $\mathrm{H}$ atoms. 


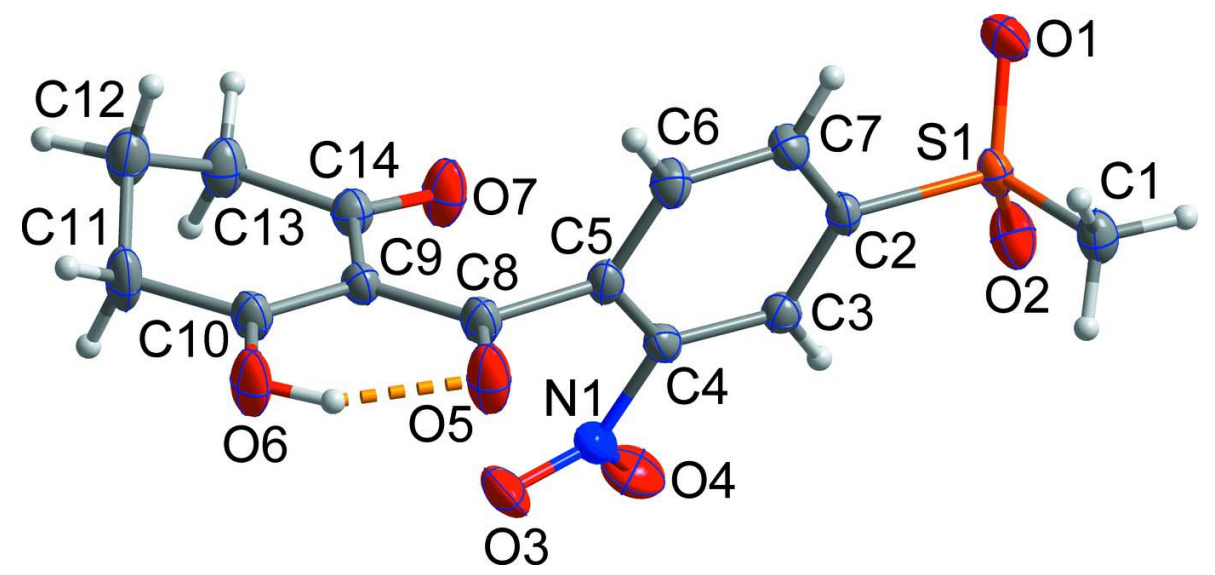

Figure 1

The molecular structure of the title compound, with atom labelling. Displacement ellipsoids are drawn at the $50 \%$ probability level. The intramolecular $\mathrm{O}-\mathrm{H}^{\cdots} \mathrm{O}$ hydrogen bond is shown as a dashed line (see Table 1 for details).

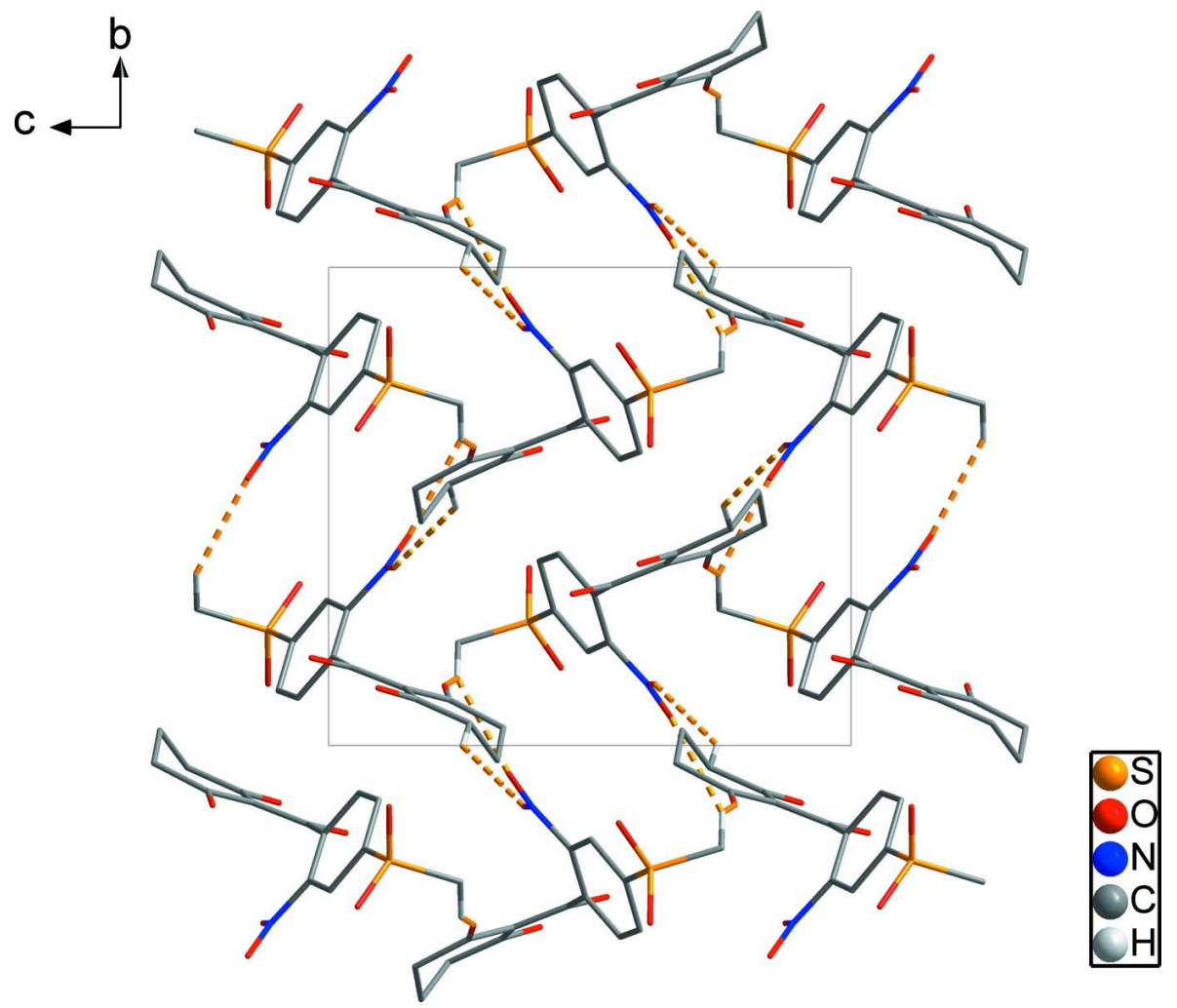

Figure 2

Crystal packing of the title compound viewed along the $a$ axis. The intermolecular $\mathrm{C}-\mathrm{H} \cdots \mathrm{O}$ hydrogen bonds are shown as dashed lines (see Table 1 for details).

3-Hydroxy-2-(4-methylsulfonyl-2-nitrobenzoyl)cyclohex-2-enone

Crystal data

$\mathrm{C}_{14} \mathrm{H}_{13} \mathrm{NO}_{7} \mathrm{~S}$

$M_{r}=339.31$

Monoclinic, $P 2{ }_{1} / c$

$a=10.4208$ (2) $\AA$ 


$$
\begin{aligned}
& b=11.2525(3) \AA \\
& c=12.3550(3) \AA \\
& \beta=95.370(1)^{\circ} \\
& V=1442.39(6) \AA^{3} \\
& Z=4 \\
& F(000)=704 \\
& D_{\mathrm{x}}=1.563 \mathrm{Mg} \mathrm{m}^{-3}
\end{aligned}
$$

\section{Data collection}

\section{Bruker APEXII CCD}

diffractometer

$\varphi$ and $\omega$ scans

Absorption correction: multi-scan

(SADABS; Bruker, 2009)

$T_{\min }=0.895, T_{\max }=0.949$

12093 measured reflections

\section{Refinement}

Refinement on $F^{2}$

Least-squares matrix: full

$R\left[F^{2}>2 \sigma\left(F^{2}\right)\right]=0.036$

$w R\left(F^{2}\right)=0.097$

$S=1.04$

2828 reflections

213 parameters

0 restraints

Primary atom site location: structure-invariant direct methods
Mo $K \alpha$ radiation, $\lambda=0.71073 \AA$

Cell parameters from 7168 reflections

$\theta=2.5-27.5^{\circ}$

$\mu=0.26 \mathrm{~mm}^{-1}$

$T=173 \mathrm{~K}$

Block, colourless

$0.43 \times 0.30 \times 0.20 \mathrm{~mm}$

2828 independent reflections

2572 reflections with $I>2 \sigma(I)$

$R_{\text {int }}=0.025$

$\theta_{\max }=26.0^{\circ}, \theta_{\min }=2.0^{\circ}$

$h=-12 \rightarrow 12$

$k=-13 \rightarrow 13$

$l=-15 \rightarrow 15$

Secondary atom site location: difference Fourier map

Hydrogen site location: mixed

$\mathrm{H}$ atoms treated by a mixture of independent and constrained refinement

$w=1 /\left[\sigma^{2}\left(F_{\mathrm{o}}^{2}\right)+(0.0538 P)^{2}+0.7458 P\right]$ where $P=\left(F_{\mathrm{o}}^{2}+2 F_{\mathrm{c}}^{2}\right) / 3$

$(\Delta / \sigma)_{\max }<0.001$

$\Delta \rho_{\max }=0.48 \mathrm{e} \AA^{-3}$

$\Delta \rho_{\min }=-0.40$ e $\AA^{-3}$

\section{Special details}

Geometry. All e.s.d.'s (except the e.s.d. in the dihedral angle between two 1.s. planes) are estimated using the full covariance matrix. The cell e.s.d.'s are taken into account individually in the estimation of e.s.d.'s in distances, angles and torsion angles; correlations between e.s.d.'s in cell parameters are only used when they are defined by crystal symmetry. An approximate (isotropic) treatment of cell e.s.d.'s is used for estimating e.s.d.'s involving 1.s. planes.

Fractional atomic coordinates and isotropic or equivalent isotropic displacement parameters $\left(\AA^{2}\right)$

\begin{tabular}{lllll}
\hline & $x$ & $y$ & $z$ & $U_{\text {iso }} * / U_{\text {eq }}$ \\
\hline S1 & $0.31056(3)$ & $0.24106(4)$ & $1.11672(3)$ & $0.02141(13)$ \\
O1 & $0.24559(11)$ & $0.12877(11)$ & $1.11593(11)$ & $0.0333(3)$ \\
O2 & $0.24978(11)$ & $0.33729(12)$ & $1.05544(10)$ & $0.0341(3)$ \\
O3 & $0.78663(13)$ & $0.37212(12)$ & $0.87472(13)$ & $0.0461(4)$ \\
O4 & $0.59220(13)$ & $0.44028(12)$ & $0.84539(11)$ & $0.0406(3)$ \\
O5 & $0.92622(11)$ & $0.18323(14)$ & $1.03272(11)$ & $0.0420(4)$ \\
O6 & $1.08825(11)$ & $0.11994(14)$ & $0.90444(11)$ & $0.0387(3)$ \\
H6O & $1.057(3)$ & $0.151(2)$ & $0.965(2)$ & $0.062(8)^{*}$ \\
O7 & $0.64741(11)$ & $0.12478(13)$ & $0.77772(11)$ & $0.0367(3)$ \\
N1 & $0.67227(14)$ & $0.37175(13)$ & $0.88940(12)$ & $0.0280(3)$ \\
C1 & $0.34825(16)$ & $0.28528(17)$ & $1.25212(13)$ & $0.0279(4)$ \\
H1A & 0.2685 & 0.2995 & 1.2863 & $0.042 *$ \\
H1B & 0.3993 & 0.3585 & 1.2540 & $0.042 *$ \\
H1C & 0.3981 & 0.2225 & 1.2918 & $0.042 *$
\end{tabular}




$\begin{array}{lllll}\text { C2 } & 0.46359(14) & 0.21719(14) & 1.06875(12) & 0.0195(3) \\ \text { C3 } & 0.51015(14) & 0.30302(14) & 1.00287(12) & 0.0206(3) \\ \text { H3 } & 0.4624 & 0.3733 & 0.9847 & 0.025^{*} \\ \text { C4 } & 0.62836(15) & 0.28346(14) & 0.96419(12) & 0.0213(3) \\ \text { C5 } & 0.70348(14) & 0.18398(15) & 0.99181(13) & 0.0224(3) \\ \text { C6 } & 0.65419(15) & 0.09958(15) & 1.05868(13) & 0.0247(3) \\ \text { H6 } & 0.7035 & 0.0309 & 1.0792 & 0.030^{*} \\ \text { C7 } & 0.53315(15) & 0.11459(14) & 1.09596(13) & 0.0234(3) \\ \text { H7 } & 0.4986 & 0.0552 & 1.1396 & 0.028^{*} \\ \text { C8 } & 0.83866(15) & 0.16470(15) & 0.95976(14) & 0.0258(4) \\ \text { C9 } & 0.86333(14) & 0.11938(14) & 0.85403(13) & 0.0221(3) \\ \text { C10 } & 0.98924(15) & 0.09727(16) & 0.83365(14) & 0.0268(4) \\ \text { C11 } & 1.02509(16) & 0.0464(2) & 0.72964(16) & 0.0378(5) \\ \text { H11A } & 1.1048 & -0.0012 & 0.7437 & 0.045^{*} \\ \text { H11B } & 1.0428 & 0.1117 & 0.6795 & 0.045^{*} \\ \text { C12 } & 0.91933(17) & -0.03099(19) & 0.67708(16) & 0.0368(4) \\ \text { H12A } & 0.9099 & -0.1027 & 0.7220 & 0.044^{*} \\ \text { H12B } & 0.9416 & -0.0569 & 0.6046 & 0.044^{*} \\ \text { C13 } & 0.79406(16) & 0.03715(17) & 0.66546(14) & 0.0312(4) \\ \text { H13A } & 0.7239 & -0.0180 & 0.6393 & 0.037^{*} \\ \text { H13B } & 0.7995 & 0.0992 & 0.6093 & 0.037^{*} \\ \text { C14 } & 0.75890(15) & 0.09568(15) & 0.76871(13) & 0.0238(3)\end{array}$

Atomic displacement parameters $\left(\AA^{2}\right)$

\begin{tabular}{lllllll}
\hline & $U^{11}$ & $U^{22}$ & $U^{33}$ & $U^{12}$ & $U^{13}$ & $U^{23}$ \\
\hline S1 & $0.0139(2)$ & $0.0295(2)$ & $0.0211(2)$ & $0.00081(14)$ & $0.00345(15)$ & $0.00020(15)$ \\
O1 & $0.0245(6)$ & $0.0388(7)$ & $0.0376(7)$ & $-0.0102(5)$ & $0.0082(5)$ & $-0.0049(6)$ \\
O2 & $0.0242(6)$ & $0.0461(8)$ & $0.0324(7)$ & $0.0129(5)$ & $0.0046(5)$ & $0.0085(6)$ \\
O3 & $0.0384(8)$ & $0.0357(8)$ & $0.0693(10)$ & $-0.0057(6)$ & $0.0325(7)$ & $0.0051(7)$ \\
O4 & $0.0450(8)$ & $0.0374(8)$ & $0.0395(8)$ & $-0.0054(6)$ & $0.0042(6)$ & $0.0145(6)$ \\
O5 & $0.0205(6)$ & $0.0733(10)$ & $0.0320(7)$ & $-0.0026(6)$ & $0.0022(5)$ & $-0.0197(7)$ \\
O6 & $0.0158(6)$ & $0.0656(10)$ & $0.0350(7)$ & $-0.0005(6)$ & $0.0033(5)$ & $-0.0182(7)$ \\
O7 & $0.0193(6)$ & $0.0557(9)$ & $0.0344(7)$ & $0.0087(5)$ & $-0.0007(5)$ & $-0.0121(6)$ \\
N1 & $0.0321(8)$ & $0.0249(7)$ & $0.0286(8)$ & $-0.0075(6)$ & $0.0116(6)$ & $-0.0025(6)$ \\
C1 & $0.0227(8)$ & $0.0394(10)$ & $0.0222(8)$ & $0.0006(7)$ & $0.0043(6)$ & $-0.0043(7)$ \\
C2 & $0.0155(7)$ & $0.0250(8)$ & $0.0185(7)$ & $-0.0002(6)$ & $0.0035(6)$ & $-0.0027(6)$ \\
C3 & $0.0196(7)$ & $0.0219(8)$ & $0.0203(7)$ & $0.0000(6)$ & $0.0022(6)$ & $-0.0026(6)$ \\
C4 & $0.0220(8)$ & $0.0236(8)$ & $0.0190(7)$ & $-0.0050(6)$ & $0.0054(6)$ & $-0.0028(6)$ \\
C5 & $0.0183(7)$ & $0.0282(9)$ & $0.0212(8)$ & $-0.0014(6)$ & $0.0039(6)$ & $-0.0074(6)$ \\
C6 & $0.0219(7)$ & $0.0267(8)$ & $0.0259(8)$ & $0.0047(6)$ & $0.0040(6)$ & $0.0005(7)$ \\
C7 & $0.0226(8)$ & $0.0252(8)$ & $0.0228(8)$ & $0.0001(6)$ & $0.0046(6)$ & $0.0027(6)$ \\
C8 & $0.0183(7)$ & $0.0318(9)$ & $0.0276(8)$ & $-0.0019(6)$ & $0.0042(6)$ & $-0.0052(7)$ \\
C9 & $0.0181(7)$ & $0.0249(8)$ & $0.0240(8)$ & $-0.0010(6)$ & $0.0051(6)$ & $-0.0029(6)$ \\
C10 & $0.0185(7)$ & $0.0340(9)$ & $0.0283(8)$ & $-0.0013(7)$ & $0.0044(6)$ & $-0.0047(7)$ \\
C11 & $0.0218(8)$ & $0.0590(13)$ & $0.0341(10)$ & $0.0007(8)$ & $0.0103(7)$ & $-0.0155(9)$ \\
C12 & $0.0304(9)$ & $0.0471(11)$ & $0.0338(10)$ & $0.0009(8)$ & $0.0082(8)$ & $-0.0131(8)$ \\
C13 & $0.0269(8)$ & $0.0437(10)$ & $0.0227(8)$ & $0.0024(7)$ & $0.0006(6)$ & $-0.0060(7)$ \\
& & & & & &
\end{tabular}


Geometric parameters $\left(A,{ }^{o}\right)$

\begin{tabular}{|c|c|c|c|}
\hline $\mathrm{S} 1-\mathrm{O} 1$ & $1.4331(13)$ & $\mathrm{C} 5-\mathrm{C} 6$ & $1.389(2)$ \\
\hline $\mathrm{S} 1-\mathrm{O} 2$ & $1.4343(12)$ & $\mathrm{C} 5-\mathrm{C} 8$ & $1.514(2)$ \\
\hline $\mathrm{S} 1-\mathrm{C} 1$ & $1.7543(17)$ & $\mathrm{C} 6-\mathrm{C} 7$ & $1.393(2)$ \\
\hline $\mathrm{S} 1-\mathrm{C} 2$ & $1.7730(15)$ & $\mathrm{C} 6-\mathrm{H} 6$ & 0.9500 \\
\hline $\mathrm{O} 3-\mathrm{N} 1$ & $1.2222(19)$ & $\mathrm{C} 7-\mathrm{H} 7$ & 0.9500 \\
\hline $\mathrm{O} 4-\mathrm{N} 1$ & $1.225(2)$ & $\mathrm{C} 8-\mathrm{C} 9$ & $1.448(2)$ \\
\hline $\mathrm{O} 5-\mathrm{C} 8$ & $1.239(2)$ & $\mathrm{C} 9-\mathrm{C} 10$ & $1.382(2)$ \\
\hline $\mathrm{O} 6-\mathrm{C} 10$ & $1.314(2)$ & $\mathrm{C} 9-\mathrm{C} 14$ & $1.467(2)$ \\
\hline $\mathrm{O} 6-\mathrm{H} 6 \mathrm{O}$ & $0.91(3)$ & $\mathrm{C} 10-\mathrm{C} 11$ & $1.486(2)$ \\
\hline $\mathrm{O} 7-\mathrm{C} 14$ & $1.222(2)$ & $\mathrm{C} 11-\mathrm{C} 12$ & $1.504(3)$ \\
\hline $\mathrm{N} 1-\mathrm{C} 4$ & $1.459(2)$ & $\mathrm{C} 11-\mathrm{H} 11 \mathrm{~A}$ & 0.9900 \\
\hline $\mathrm{C} 1-\mathrm{H} 1 \mathrm{~A}$ & 0.9800 & C11-H11B & 0.9900 \\
\hline $\mathrm{C} 1-\mathrm{H} 1 \mathrm{~B}$ & 0.9800 & $\mathrm{C} 12-\mathrm{C} 13$ & $1.509(2)$ \\
\hline $\mathrm{C} 1-\mathrm{H} 1 \mathrm{C}$ & 0.9800 & $\mathrm{C} 12-\mathrm{H} 12 \mathrm{~A}$ & 0.9900 \\
\hline $\mathrm{C} 2-\mathrm{C} 3$ & $1.380(2)$ & $\mathrm{C} 12-\mathrm{H} 12 \mathrm{~B}$ & 0.9900 \\
\hline $\mathrm{C} 2-\mathrm{C} 7$ & $1.388(2)$ & $\mathrm{C} 13-\mathrm{C} 14$ & $1.511(2)$ \\
\hline $\mathrm{C} 3-\mathrm{C} 4$ & $1.380(2)$ & $\mathrm{C} 13-\mathrm{H} 13 \mathrm{~A}$ & 0.9900 \\
\hline $\mathrm{C} 3-\mathrm{H} 3$ & 0.9500 & C13-H13B & 0.9900 \\
\hline $\mathrm{C} 4-\mathrm{C} 5$ & $1.390(2)$ & & \\
\hline $\mathrm{O} 1-\mathrm{S} 1-\mathrm{O} 2$ & $118.50(8)$ & $\mathrm{C} 2-\mathrm{C} 7-\mathrm{H} 7$ & 120.4 \\
\hline $\mathrm{O} 1-\mathrm{S} 1-\mathrm{C} 1$ & $108.66(8)$ & $\mathrm{C} 6-\mathrm{C} 7-\mathrm{H} 7$ & 120.4 \\
\hline $\mathrm{O} 2-\mathrm{S} 1-\mathrm{C} 1$ & $109.73(8)$ & $\mathrm{O} 5-\mathrm{C} 8-\mathrm{C} 9$ & $122.34(14)$ \\
\hline $\mathrm{O} 1-\mathrm{S} 1-\mathrm{C} 2$ & $107.66(7)$ & $\mathrm{O} 5-\mathrm{C} 8-\mathrm{C} 5$ & $115.18(14)$ \\
\hline $\mathrm{O} 2-\mathrm{S} 1-\mathrm{C} 2$ & $107.73(7)$ & $\mathrm{C} 9-\mathrm{C} 8-\mathrm{C} 5$ & $122.32(13)$ \\
\hline $\mathrm{C} 1-\mathrm{S} 1-\mathrm{C} 2$ & $103.51(7)$ & $\mathrm{C} 10-\mathrm{C} 9-\mathrm{C} 8$ & $118.67(14)$ \\
\hline $\mathrm{C} 10-\mathrm{O} 6-\mathrm{H} 6 \mathrm{O}$ & $107.8(17)$ & $\mathrm{C} 10-\mathrm{C} 9-\mathrm{C} 14$ & $119.31(14)$ \\
\hline $\mathrm{O} 3-\mathrm{N} 1-\mathrm{O} 4$ & $124.40(15)$ & $\mathrm{C} 8-\mathrm{C} 9-\mathrm{C} 14$ & $122.01(14)$ \\
\hline $\mathrm{O} 3-\mathrm{N} 1-\mathrm{C} 4$ & $117.66(15)$ & $\mathrm{O} 6-\mathrm{C} 10-\mathrm{C} 9$ & $122.94(15)$ \\
\hline $\mathrm{O} 4-\mathrm{N} 1-\mathrm{C} 4$ & $117.94(14)$ & $\mathrm{O} 6-\mathrm{C} 10-\mathrm{C} 11$ & $113.90(14)$ \\
\hline $\mathrm{S} 1-\mathrm{C} 1-\mathrm{H} 1 \mathrm{~A}$ & 109.5 & $\mathrm{C} 9-\mathrm{C} 10-\mathrm{C} 11$ & $123.15(15)$ \\
\hline $\mathrm{S} 1-\mathrm{C} 1-\mathrm{H} 1 \mathrm{~B}$ & 109.5 & $\mathrm{C} 10-\mathrm{C} 11-\mathrm{C} 12$ & $111.30(14)$ \\
\hline $\mathrm{H} 1 \mathrm{~A}-\mathrm{C} 1-\mathrm{H} 1 \mathrm{~B}$ & 109.5 & $\mathrm{C} 10-\mathrm{C} 11-\mathrm{H} 11 \mathrm{~A}$ & 109.4 \\
\hline $\mathrm{S} 1-\mathrm{C} 1-\mathrm{H} 1 \mathrm{C}$ & 109.5 & $\mathrm{C} 12-\mathrm{C} 11-\mathrm{H} 11 \mathrm{~A}$ & 109.4 \\
\hline $\mathrm{H} 1 \mathrm{~A}-\mathrm{C} 1-\mathrm{H} 1 \mathrm{C}$ & 109.5 & $\mathrm{C} 10-\mathrm{C} 11-\mathrm{H} 11 \mathrm{~B}$ & 109.4 \\
\hline $\mathrm{H} 1 \mathrm{~B}-\mathrm{C} 1-\mathrm{H} 1 \mathrm{C}$ & 109.5 & $\mathrm{C} 12-\mathrm{C} 11-\mathrm{H} 11 \mathrm{~B}$ & 109.4 \\
\hline $\mathrm{C} 3-\mathrm{C} 2-\mathrm{C} 7$ & $121.38(14)$ & $\mathrm{H} 11 \mathrm{~A}-\mathrm{C} 11-\mathrm{H} 11 \mathrm{~B}$ & 108.0 \\
\hline $\mathrm{C} 3-\mathrm{C} 2-\mathrm{S} 1$ & $117.90(12)$ & $\mathrm{C} 11-\mathrm{C} 12-\mathrm{C} 13$ & $109.84(16)$ \\
\hline $\mathrm{C} 7-\mathrm{C} 2-\mathrm{S} 1$ & $120.72(12)$ & $\mathrm{C} 11-\mathrm{C} 12-\mathrm{H} 12 \mathrm{~A}$ & 109.7 \\
\hline $\mathrm{C} 2-\mathrm{C} 3-\mathrm{C} 4$ & $117.84(14)$ & $\mathrm{C} 13-\mathrm{C} 12-\mathrm{H} 12 \mathrm{~A}$ & 109.7 \\
\hline $\mathrm{C} 2-\mathrm{C} 3-\mathrm{H} 3$ & 121.1 & $\mathrm{C} 11-\mathrm{C} 12-\mathrm{H} 12 \mathrm{~B}$ & 109.7 \\
\hline $\mathrm{C} 4-\mathrm{C} 3-\mathrm{H} 3$ & 121.1 & $\mathrm{C} 13-\mathrm{C} 12-\mathrm{H} 12 \mathrm{~B}$ & 109.7 \\
\hline $\mathrm{C} 3-\mathrm{C} 4-\mathrm{C} 5$ & $122.95(14)$ & $\mathrm{H} 12 \mathrm{~A}-\mathrm{C} 12-\mathrm{H} 12 \mathrm{~B}$ & 108.2 \\
\hline $\mathrm{C} 3-\mathrm{C} 4-\mathrm{N} 1$ & $116.98(14)$ & $\mathrm{C} 12-\mathrm{C} 13-\mathrm{C} 14$ & $114.66(14)$ \\
\hline
\end{tabular}




\begin{tabular}{|c|c|c|c|}
\hline $\mathrm{C} 5-\mathrm{C} 4-\mathrm{N} 1$ & $120.06(14)$ & $\mathrm{C} 12-\mathrm{C} 13-\mathrm{H} 13 \mathrm{~A}$ & 108.6 \\
\hline $\mathrm{C} 6-\mathrm{C} 5-\mathrm{C} 4$ & $117.75(14)$ & $\mathrm{C} 14-\mathrm{C} 13-\mathrm{H} 13 \mathrm{~A}$ & 108.6 \\
\hline $\mathrm{C} 6-\mathrm{C} 5-\mathrm{C} 8$ & $117.56(14)$ & $\mathrm{C} 12-\mathrm{C} 13-\mathrm{H} 13 \mathrm{~B}$ & 108.6 \\
\hline $\mathrm{C} 4-\mathrm{C} 5-\mathrm{C} 8$ & $124.58(15)$ & $\mathrm{C} 14-\mathrm{C} 13-\mathrm{H} 13 \mathrm{~B}$ & 108.6 \\
\hline $\mathrm{C} 5-\mathrm{C} 6-\mathrm{C} 7$ & $120.71(15)$ & $\mathrm{H} 13 \mathrm{~A}-\mathrm{C} 13-\mathrm{H} 13 \mathrm{~B}$ & 107.6 \\
\hline $\mathrm{C} 5-\mathrm{C} 6-\mathrm{H} 6$ & 119.6 & $\mathrm{O} 7-\mathrm{C} 14-\mathrm{C} 9$ & $122.24(15)$ \\
\hline $\mathrm{C} 7-\mathrm{C} 6-\mathrm{H} 6$ & 119.6 & $\mathrm{O} 7-\mathrm{C} 14-\mathrm{C} 13$ & $120.09(14)$ \\
\hline $\mathrm{C} 2-\mathrm{C} 7-\mathrm{C} 6$ & $119.30(15)$ & $\mathrm{C} 9-\mathrm{C} 14-\mathrm{C} 13$ & $117.63(14)$ \\
\hline $\mathrm{O} 1-\mathrm{S} 1-\mathrm{C} 2-\mathrm{C} 3$ & $141.94(12)$ & $\mathrm{C} 6-\mathrm{C} 5-\mathrm{C} 8-\mathrm{O} 5$ & $-73.6(2)$ \\
\hline $\mathrm{O} 2-\mathrm{S} 1-\mathrm{C} 2-\mathrm{C} 3$ & $13.08(15)$ & $\mathrm{C} 4-\mathrm{C} 5-\mathrm{C} 8-\mathrm{O} 5$ & $102.5(2)$ \\
\hline $\mathrm{C} 1-\mathrm{S} 1-\mathrm{C} 2-\mathrm{C} 3$ & $-103.11(13)$ & $\mathrm{C} 6-\mathrm{C} 5-\mathrm{C} 8-\mathrm{C} 9$ & $101.98(19)$ \\
\hline $\mathrm{O} 1-\mathrm{S} 1-\mathrm{C} 2-\mathrm{C} 7$ & $-37.36(15)$ & $\mathrm{C} 4-\mathrm{C} 5-\mathrm{C} 8-\mathrm{C} 9$ & $-81.9(2)$ \\
\hline $\mathrm{O} 2-\mathrm{S} 1-\mathrm{C} 2-\mathrm{C} 7$ & $-166.22(13)$ & $\mathrm{O} 5-\mathrm{C} 8-\mathrm{C} 9-\mathrm{C} 10$ & $-0.7(3)$ \\
\hline $\mathrm{C} 1-\mathrm{S} 1-\mathrm{C} 2-\mathrm{C} 7$ & $77.59(15)$ & $\mathrm{C} 5-\mathrm{C} 8-\mathrm{C} 9-\mathrm{C} 10$ & $-175.97(15)$ \\
\hline $\mathrm{C} 7-\mathrm{C} 2-\mathrm{C} 3-\mathrm{C} 4$ & $0.3(2)$ & $\mathrm{O} 5-\mathrm{C} 8-\mathrm{C} 9-\mathrm{C} 14$ & $178.90(17)$ \\
\hline $\mathrm{S} 1-\mathrm{C} 2-\mathrm{C} 3-\mathrm{C} 4$ & $-179.00(11)$ & $\mathrm{C} 5-\mathrm{C} 8-\mathrm{C} 9-\mathrm{C} 14$ & $3.6(3)$ \\
\hline $\mathrm{C} 2-\mathrm{C} 3-\mathrm{C} 4-\mathrm{C} 5$ & $-2.4(2)$ & $\mathrm{C} 8-\mathrm{C} 9-\mathrm{C} 10-\mathrm{O} 6$ & $-2.8(3)$ \\
\hline $\mathrm{C} 2-\mathrm{C} 3-\mathrm{C} 4-\mathrm{N} 1$ & $176.38(13)$ & $\mathrm{C} 14-\mathrm{C} 9-\mathrm{C} 10-\mathrm{O} 6$ & $177.62(16)$ \\
\hline $\mathrm{O} 3-\mathrm{N} 1-\mathrm{C} 4-\mathrm{C} 3$ & $162.96(15)$ & $\mathrm{C} 8-\mathrm{C} 9-\mathrm{C} 10-\mathrm{C} 11$ & $177.56(17)$ \\
\hline $\mathrm{O} 4-\mathrm{N} 1-\mathrm{C} 4-\mathrm{C} 3$ & $-17.2(2)$ & $\mathrm{C} 14-\mathrm{C} 9-\mathrm{C} 10-\mathrm{C} 11$ & $-2.0(3)$ \\
\hline $\mathrm{O} 3-\mathrm{N} 1-\mathrm{C} 4-\mathrm{C} 5$ & $-18.3(2)$ & $\mathrm{O} 6-\mathrm{C} 10-\mathrm{C} 11-\mathrm{C} 12$ & $151.87(17)$ \\
\hline $\mathrm{O} 4-\mathrm{N} 1-\mathrm{C} 4-\mathrm{C} 5$ & $161.57(15)$ & $\mathrm{C} 9-\mathrm{C} 10-\mathrm{C} 11-\mathrm{C} 12$ & $-28.4(3)$ \\
\hline $\mathrm{C} 3-\mathrm{C} 4-\mathrm{C} 5-\mathrm{C} 6$ & $2.0(2)$ & $\mathrm{C} 10-\mathrm{C} 11-\mathrm{C} 12-\mathrm{C} 13$ & $53.4(2)$ \\
\hline $\mathrm{N} 1-\mathrm{C} 4-\mathrm{C} 5-\mathrm{C} 6$ & $-176.65(14)$ & $\mathrm{C} 11-\mathrm{C} 12-\mathrm{C} 13-\mathrm{C} 14$ & $-51.4(2)$ \\
\hline $\mathrm{C} 3-\mathrm{C} 4-\mathrm{C} 5-\mathrm{C} 8$ & $-174.05(14)$ & $\mathrm{C} 10-\mathrm{C} 9-\mathrm{C} 14-\mathrm{O} 7$ & $-172.27(17)$ \\
\hline $\mathrm{N} 1-\mathrm{C} 4-\mathrm{C} 5-\mathrm{C} 8$ & $7.3(2)$ & $\mathrm{C} 8-\mathrm{C} 9-\mathrm{C} 14-\mathrm{O} 7$ & $8.1(3)$ \\
\hline $\mathrm{C} 4-\mathrm{C} 5-\mathrm{C} 6-\mathrm{C} 7$ & $0.3(2)$ & $\mathrm{C} 10-\mathrm{C} 9-\mathrm{C} 14-\mathrm{C} 13$ & $5.5(2)$ \\
\hline $\mathrm{C} 8-\mathrm{C} 5-\mathrm{C} 6-\mathrm{C} 7$ & $176.70(14)$ & $\mathrm{C} 8-\mathrm{C} 9-\mathrm{C} 14-\mathrm{C} 13$ & $-174.07(16)$ \\
\hline $\mathrm{C} 3-\mathrm{C} 2-\mathrm{C} 7-\mathrm{C} 6$ & $2.0(2)$ & $\mathrm{C} 12-\mathrm{C} 13-\mathrm{C} 14-\mathrm{O} 7$ & $-160.12(17)$ \\
\hline $\mathrm{S} 1-\mathrm{C} 2-\mathrm{C} 7-\mathrm{C} 6$ & $-178.77(12)$ & $\mathrm{C} 12-\mathrm{C} 13-\mathrm{C} 14-\mathrm{C} 9$ & $22.0(2)$ \\
\hline $\mathrm{C} 5-\mathrm{C} 6-\mathrm{C} 7-\mathrm{C} 2$ & $-2.3(2)$ & & \\
\hline
\end{tabular}

Hydrogen-bond geometry $\left(A,{ }^{\circ}\right)$

\begin{tabular}{lllll}
\hline$D-\mathrm{H} \cdots A$ & $D-\mathrm{H}$ & $\mathrm{H} \cdots A$ & $D \cdots A$ & $D-\mathrm{H} \cdots A$ \\
\hline $\mathrm{O} 6-\mathrm{H} 6 O \cdots \mathrm{O} 5$ & $0.91(3)$ & $1.71(3)$ & $2.524(2)$ & $148(2)$ \\
$\mathrm{C} 1-\mathrm{H} 1 B \cdots \mathrm{O} 4{ }^{\mathrm{i}}$ & 0.98 & 2.58 & $3.393(2)$ & 140 \\
$\mathrm{C} 1-\mathrm{H} 1 B \cdots \mathrm{O} 7^{\mathrm{ii}}$ & 0.98 & 2.58 & $3.265(2)$ & 127 \\
$\mathrm{C} 11-\mathrm{H} 11 A \cdots \mathrm{O} 3^{\mathrm{iii}}$ & 0.99 & 2.40 & $3.135(2)$ & 131 \\
\hline
\end{tabular}

Symmetry codes: (i) $-x+1,-y+1,-z+2$; (ii) $x,-y+1 / 2, z+1 / 2$; (iii) $-x+2, y-1 / 2,-z+3 / 2$. 